\title{
Wavelet transforms for functions with values in Lebesgue spaces
}

\author{
Cornelia Kaiser $^{a}$ \\ ${ }^{a}$ Universität Karlsruhe, Englerstraße 2, 76128 Karlsruhe, Germany
}

\begin{abstract}
We study the continuous and semi-discrete wavelet transform applied to functions with values in Lebesgue spaces.

Keywords: Continuous wavelet transform, semi-discrete wavelet transform, Littlewood-Paley estimates, vectorvalued harmonic analysis
\end{abstract}

\section{INTRODUCTION}

In this paper we study the continuous and semi-discrete wavelet transform for functions with values in Lebesgue spaces. What we basically will show is that the classical scalar-valued results carry over to this vector-valued setting. In this sense our results can be considered as an extension and continuation of the work of T. Figiel. ${ }^{1}$ In the late 80 's, he proved that the scalar-valued theory on orthonormal wavelets can be transfered to the vectorvalued setting precisely when the underlying Banach space has the UMD property. (Most reflexive Banach spaces satisfy this condition.) $\mathrm{We}^{2}$ extended the Figiel result to redundant discrete wavelet transforms (wavelet frames) and proved boundedness of localization operators with operator-valued symbol connected with the discrete wavelet transform.

Here we focus on the continuous and the semi-discrete wavelet transform, for which we prove a norm estimate and a reconstruction formula corresponding to the classical scalar-valued Littlewood-Paley theory (see e.g. [3]). Our results are also related to square function estimates from [4] and the homogeneous Triebel-Lizorkin spaces (see e.g. [5]).

Our primary interest is from a theoretical point of view. However, a vector-valued wavelet theory should also be useful in applications, e.g. to the study of differential operators. In fact, the methods we use have already been successfully applied in various fields (regularity of solutions of partial differential equations, ${ }^{6}$ functional calculus, ${ }^{7}$ stochastic integration $\left.{ }^{8}\right)$.

\section{DEFINITIONS, NOTATIONS, AND MAIN TOOLS}

By $\mathcal{B}(X, Y)$ we denote the space of bounded linear operators from a Banach space $X$ to some Banach space $Y$ and $X^{\prime}=\mathcal{B}(X, \mathbb{C})$ is the dual space of $X$. If $x^{\prime} \in X^{\prime}$ and $x \in X$, we also write $\left\langle x^{\prime}, x\right\rangle_{X}$ for $x^{\prime}(x)$.

The Schwartz class $\mathcal{S}\left(\mathbb{R}^{N}, X\right)$ is the space of $X$-valued rapidly decreasing smooth functions on $\mathbb{R}^{N}$.

$\mathbb{N}=\{1,2, \ldots\}$ is the set of natural numbers and $\mathbb{N}_{0}=\{0\} \cup \mathbb{N}$. If $r$ is a positive real number, then $[r]=$ $\max \left\{n \in \mathbb{N}_{0}: n \leq r\right\}$. By $R_{*}$ we denote the multiplicative group $\mathbb{R}_{+}$, endowed with its invariant measure $\frac{d t}{t}$.

cornelia.kaiser@math.uni-karlsruhe.de Copyright 2005 Society of Photo-Optical Instrumentation Engineers.

This paper will be published in Proceedings of SPIE Vol. 5914: Wavelets XI (SPIE, Bellingham, WA, 2005) and is made available as an electronic preprint with permission of SPIE. One print or electronic copy may be made for personal use only. Systematic or multiple reproduction, distribution to multiple locations via electronic or other means, duplication of any material in this paper for a fee or for commercial purposes, or modification of the content of the paper are prohibited. 


\subsection{Some notions from Banach space theory}

We will need two notions from the theory of Banach spaces, the notion of Fourier type and the so-called UMD property.

A Banach space $X$ has Fourier type $r \in[1,2]$ if the Fourier transform $\mathcal{F}$ defined by

$$
(\mathcal{F} f)(u)=\widehat{f}(u)=(2 \pi)^{-N / 2} \int_{\mathbb{R}^{N}} e^{-i u v} f(v) d v, \quad f \in \mathcal{S}(\mathbb{R}, X),
$$

extends to a bounded linear operator from $L_{r}(\mathbb{R}, X)$ to $L_{r^{\prime}}(\mathbb{R}, X)$, where $\frac{1}{r}+\frac{1}{r^{\prime}}=1$.

The notion of Fourier type was introduced by Peetre. ${ }^{9}$ Each Banach space has Fourier type 1 and the notion becomes more restrictive as $p$ increases to 2 . A Banach space has Fourier type 2 if and only if it is isomorphic to a Hilbert space. ${ }^{10}$ The dual space and each closed subspace of a Banach space $X$ has the same Fourier type as $X$.

A Banach space $X$ is a $U M D$ space if and only if the Hilbert transform

$$
(\mathcal{H} f)(u)=\mathrm{PV}-\int \frac{f(v)}{u-v} d v, \quad f \in \mathcal{S}(\mathbb{R}, X),
$$

extends to a bounded linear operator on $L_{p}(\mathbb{R}, X)$ for some (and thus for each) $p \in(1, \infty)$.

There are several equivalent definitions of UMD spaces (see [11, p.141-142] and the references given there). One of these equivalent definitions is the property of unconditionality of martingale differences, where the abbreviation UMD comes from.

It is clear from our definition that each Hilbert space is a UMD space. The dual space and each closed subspace of a UMD space is a UMD space. A UMD space $X$ always has a uniformly convex renorming ${ }^{12}$ and therefore is super-reflexive. ${ }^{13}$ In particular, $\ell_{1}$ is not finitely representable in $X$. Hence $X$ is B-convex $[14$, Theorem 13.6$]$. Now it follows from a result from Bourgain ${ }^{15,16}$ that every UMD-space has some Fourier type $r>1$.

\subsection{The Lebesgue-Bochner spaces}

In this paper we will deal with a special class of Banach spaces, the Lebesgue-Bochner spaces. For convenience we shortly recall their definition here. For more details we refer to [17].

Let $(\Omega, \Sigma, \mu)$ be a $\sigma$-finite measure space and let $X$ be a Banach space. For a $\mu$-measurable function $f: \Omega \rightarrow X$ we write

$$
\begin{gathered}
\|f\|_{L_{p}(\Omega, \mu, X)}=\left(\int_{\Omega}\|f(\omega)\|_{X}^{p} d \mu(\omega)\right)^{1 / p}, \quad p \in[1, \infty), \\
\|f\|_{L_{\infty}(\Omega, \mu, X)}=\operatorname{ess} \sup \left\{\|f(\omega)\|_{X}: \omega \in \Omega\right\} .
\end{gathered}
$$

Now the symbol $L_{p}(\Omega, \mu, X)$ stands for the set of all (equivalence classes of) $\mu$-measurable functions $f: \Omega \rightarrow X$ such that $\|f\|_{L_{p}(\Omega, \mu, X)}$ is finite. Normed by $\|\cdot\|_{L_{p}(\Omega, \mu, X)}$, the vector space $L_{p}(\Omega, \mu)$ becomes a Banach space. If $X=\mathbb{C}$, we write $L_{p}(\Omega, \mu)$ for $L_{p}(\Omega, \mu, \mathbb{C})$.

If the dual space $X^{\prime}$ of $X$ has the Radon-Nikodym property (in particular, if $X$ is reflexive) and $p \in[1, \infty$ ), then the dual space of $L_{p}(\Omega, \mu, X)$ is isometrically isomorphic to $L_{p^{\prime}}\left(\Omega, \mu, X^{\prime}\right)$, where $p^{\prime}$ is the dual index of $p$, i.e. $\frac{1}{p}+\frac{1}{p^{\prime}}=1 .^{17}$

If $X$ has Fourier type $r$, then $L_{p}(\Omega, \mu, X)$ has Fourier type $\min \left\{r, p, p^{\prime}\right\} .{ }^{18}$ If $X$ is a UMD space and $p \in(1, \infty)$, then $L_{p}(\Omega, \mu, X)$ is a UMD space [11,p.145]. 


\subsection{R-boundedness}

For the notion of R-boundedness we need the Rademacher functions, defined by

$$
r_{k}(t)=\operatorname{sign} \sin \left(2^{k} \pi t\right), \quad t \in[0,1], k \in \mathbb{N} .
$$

The sequence $\left(r_{k}\right)$ is orthonormal in $L_{2}([0,1])$, as one can easily see from their graphs:
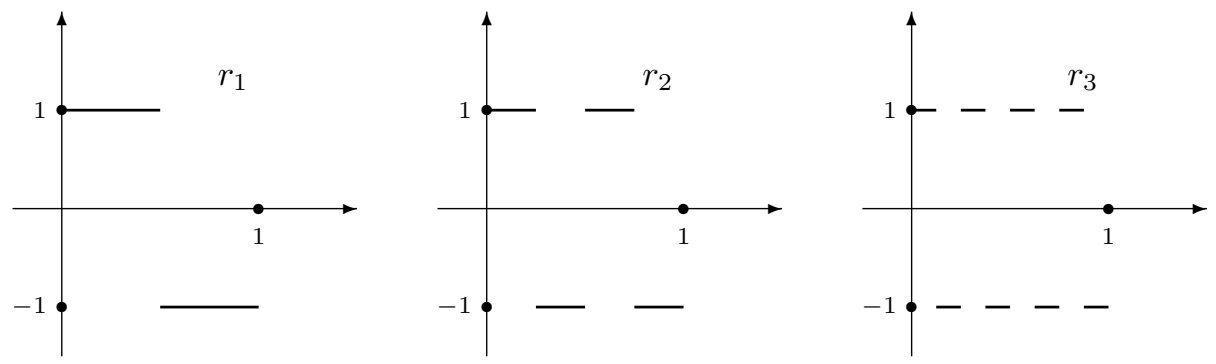

In the theory of Rademacher sums there is a fundamental principle, the so-called contraction principle.

Lemma 2.1 (CONTRACTION PRINCIPLE). Let $X$ be a Banach space. Then for each $n \in \mathbb{N}$, all complex numbers $a_{1}, \ldots, a_{n}$ and all $x_{1}, \ldots, x_{n} \in X$,

$$
\left\|\sum_{k=1}^{n} a_{k} r_{k} x_{k}\right\|_{L_{2}([0,1], X)} \leq 2 \max _{1 \leq k \leq n}\left|a_{k}\right|\left\|\sum_{k=1}^{n} r_{k} x_{k}\right\|_{L_{2}([0,1], X)}
$$

The contraction principle basically says that one can "drop out" scalars from Rademacher sums. If $X$ is a Hilbert space, then the same is true if we replace the scalars $a_{k}$ by bounded linear operators $T_{k}$ on $X$. (One only has to replace the absolute value $\left|a_{k}\right|$ by the operator norm $\left\|T_{k}\right\|$.) But if $X$ is an arbitrary Banach space this does not hold in general. So we make the following definition.

Definition 2.2. Let $X$ and $Y$ be Banach spaces. A set of operators $\tau \subseteq \mathcal{B}(X, Y)$ is called R-bounded if there is a constant $C<\infty$ such that for all $n \in \mathbb{N}$, all $T_{1}, \ldots, T_{m} \in \tau$ and all $x_{1}, \ldots x_{m} \in X$ we have that

$$
\left\|\sum_{k=1}^{n} r_{k} T_{k} x_{k}\right\|_{L_{2}([0,1], Y)} \leq C\left\|\sum_{k=1}^{n} r_{k} x_{k}\right\|_{L_{2}([0,1], X)} .
$$

The infimum over all $C$ such that (1) holds is called the R-bound of $\tau$ and is denoted by $R(\tau)$.

If $X$ and $Y$ are Hilbert spaces, then $\tau$ is R-bounded if and only if $\tau$ is uniformly bounded with respect to the operator norm in $\mathcal{B}(X, Y)$. It turns out that in many situations R-boundedness is the right notion for generalizing Hilbert space results to Banach spaces. On of these situations is the operator-valued Fourier multiplier theorem below.

We also recall a classical inequality from the theory of Rademacher sums, which will be very useful in the proof of our main result on the continuous wavelet transform.

LEMma 2.3 (Kahane's INEQUALITy). For $q \in[1, \infty)$, there is a constant $K_{q}<\infty$ such that for each Banach space $X$, each $n \in \mathbb{N}$ and all $x_{1}, \ldots, x_{n} \in X$,

$$
\frac{1}{K_{q}}\left\|\sum_{k=1}^{n} r_{k} x_{k}\right\|_{L_{2}([0,1], X)} \leq\left\|\sum_{k=1}^{n} r_{k} x_{k}\right\|_{L_{q}([0,1], X)} \leq K_{q}\left\|\sum_{k=1}^{n} r_{k} x_{k}\right\|_{L_{2}([0,1], X)} .
$$

In the case that $X$ is a Hilbert space with scalar product $\langle\cdot \mid \cdot\rangle$, we have that

$$
\left\|\sum_{k=1}^{n} r_{k} x_{k}\right\|_{L_{2}([0,1], X)}^{2}=\int_{0}^{1}\left\langle\sum_{k=1}^{n} r_{k}(t) x_{k} \mid \sum_{j=1}^{n} r_{j}(t) x_{j}\right\rangle d t=\sum_{k=1}^{n} \sum_{j=1}^{n}\left\langle x_{k} \mid x_{j}\right\rangle \int_{0}^{1} r_{k}(t) r_{j}(t) d t=\sum_{k=1}^{n}\left\|x_{k}\right\|_{X}^{2} .
$$


Hence in this special case Kahane's inequality reads

$$
\frac{1}{K_{q}}\left(\sum_{k=1}^{n}\left\|x_{k}\right\|_{X}^{2}\right)^{1 / 2} \leq\left\|\sum_{k=1}^{n} r_{k} x_{k}\right\|_{L_{q}([0,1], X)} \leq K_{q}\left(\sum_{k=1}^{n}\left\|x_{k}\right\|_{X}^{2}\right)^{1 / 2}
$$

\subsection{An operator-valued Fourier multiplier theorem of Mihlin type}

Here we will state the main tool we will use in the proof of our result on the continuous wavelet transform. For this we first make the following definition.

Definition 2.4. Let $X, Y$ be Banach spaces, $p \in(1, \infty)$, and $M: \mathbb{R}^{N} \backslash\{0\} \rightarrow \mathcal{B}(X, Y)$ be a bounded measurable function. For $f \in \mathcal{S}\left(\mathbb{R}^{N}, X\right)$ consider

$$
T f:=\mathcal{F}^{-1}[M(\cdot) \widehat{f}(\cdot)] \in L_{\infty}\left(\mathbb{R}^{N}, Y\right) .
$$

Then $M$ is a Fourier multiplier from $L_{p}\left(\mathbb{R}^{N}, X\right)$ to $L_{p}\left(\mathbb{R}^{N}, Y\right)$ provided there is a constant $C_{p}<\infty$ so that

$$
\|T f\|_{L_{p}\left(\mathbb{R}^{N}, Y\right)} \leq C_{p}\|f\|_{L_{p}\left(\mathbb{R}^{N}, X\right)}
$$

for each $f \in \mathcal{S}\left(\mathbb{R}^{N}, X\right)$. The unique extension of $T$ to an operator in $\mathcal{B}\left(L_{p}\left(\mathbb{R}^{N}, X\right), L_{p}\left(\mathbb{R}^{N}, Y\right)\right)$ is also denoted by $T$.

With this definition the following Mihlin-type fourier multiplier theorem holds. For a proof see [19].

TheOREM 2.5. Let $X$ and $Y$ be UMD spaces with Fourier type $r \in(1,2]$, let $N \in \mathbb{N}$, and $l=\left[\frac{N}{r}\right]+1$. Assume that $M: \mathbb{R}^{N} \backslash\{0\} \rightarrow \mathcal{B}(X, Y)$ is measurable, the distributional derivatives $D^{\alpha} M$ are represented by measurable functions for each $\alpha \in \mathbb{N}_{0}^{N}$ with $|\alpha| \leq l$, and the set

$$
\tau=\left\{|\xi|^{|\alpha|}\left(D^{\alpha} M\right)(\xi): \xi \in \mathbb{R}^{N} \backslash\{0\},|\alpha| \leq l\right\}
$$

is $R$-bounded. Then $M$ is a Fourier multiplier from $L_{p}\left(\mathbb{R}^{N}, X\right)$ to $L_{p}\left(\mathbb{R}^{N}, Y\right)$ for $p \in(1, \infty)$.

If $X$ and $Y$ are Hilbert spaces, then R-boundedness reduces to boundedness. Therefore Theorem 2.5 can be seen as a generalization of a corresponding result in the Hilbert space case by Schwartz. ${ }^{20}$

REMARK 2.6. Suppose the conditions of Theorem 2.5 are satisfied. Then $[M(\cdot)]^{\prime}: \mathbb{R}^{N} \backslash\{0\} \rightarrow \mathcal{B}\left(Y^{\prime}, X^{\prime}\right)$ also satisfies the conditions of Theorem 2.5 (cf. e.g. [21, Theorem 2.2.14]).

\section{THE CONTINUOUS WAVELET TRANSFORM}

In this section we assume that $(\Omega, \Sigma, \mu)$ is some $\sigma$-finite measure space and $q \in(1, \infty)$. By $X$ we denote the Lebesgue space $L_{q}(\Omega, \mu)$. Then $X$ is a UMD space with Fourier type $r:=\min \left\{q, q^{\prime}\right\}$. Furthermore, let $N \in \mathbb{N}$.

We will show that the continuous wavelet transform is bounded from $L_{p}\left(\mathbb{R}^{N}, X\right)$ to $L_{p}\left(\mathbb{R}^{N}, Y\right)$, where we will choose $Y=L_{q}\left(\Omega, \mu, L_{2}\left(\mathbb{R}_{*}\right)\right)$. For this we need the following two assumptions on the wavelet $\psi \in L_{2}\left(\mathbb{R}^{N}\right)$ :

(C1) For all $\alpha \in \mathbb{N}_{0}^{N}$ with $|\alpha| \leq l:=\left[\frac{N}{r}\right]+1$, the distributional derivatives $D^{\alpha} \widehat{\psi}$ are represented by measurable functions and

$$
C_{\alpha}(\psi):=\sup _{|\xi|=1}\left(\int_{0}^{\infty} t^{2|\alpha|}\left|\left(D^{\alpha} \widehat{\psi}\right)(t \xi)\right|^{2} \frac{d t}{t}\right)^{1 / 2}<\infty
$$

(C2) $c_{0}(\psi):=\inf _{|\xi|=1}\left(\int_{0}^{\infty}|\widehat{\psi}(t \xi)|^{2} \frac{d t}{t}\right)^{1 / 2}>0$. 
Now we define the continuous wavelet transform, applied to some smooth $X$-valued function. For $t \in \mathbb{R} \backslash\{0\}$ we write $\psi_{t}$ for the function defined by $\psi_{t}(s)=|t|^{-N} \psi\left(t^{-1} s\right), s \in \mathbb{R}^{N}$.

Definition 3.1. The continuous wavelet transform $\mathcal{W}_{\psi} f$ of a function $f \in \mathcal{S}\left(\mathbb{R}^{N}, X\right)$ with respect to the wavelet $\psi$ is given by

$$
\left(\mathcal{W}_{\psi} f\right)(t, s):=\left(\psi_{t} * f\right)(s)=\int_{\mathbb{R}^{N}} t^{-N} \psi\left(\frac{s-u}{t}\right) f(u) d u, \quad t>0, s \in \mathbb{R}^{N} .
$$

First we will prove that the continuous wavelet transform can be extended to a bounded linear operator from $L_{p}\left(\mathbb{R}^{N}, X\right)$ to $L_{p}\left(\mathbb{R}^{N}, Y\right)$. More precisely, we show that the following theorem holds.

Theorem 3.2. Let $(\Omega, \Sigma, \mu)$ be a $\sigma$-finite measure space, $q \in(1, \infty)$, and $X=L_{q}(\Omega, \mu)$. If $p \in(1, \infty)$ and $\psi \in L_{2}\left(\mathbb{R}^{N}\right)$ satisfies $(\mathrm{C} 1)$, then there is a constant $C$ such that for all $f \in \mathcal{S}\left(\mathbb{R}^{N}, X\right)$,

$$
\left\|\left(\int_{0}^{\infty}\left|\left(\mathcal{W}_{\psi} f\right)(t, \cdot)\right|^{2} \frac{d t}{t}\right)^{1 / 2}\right\|_{L_{p}\left(\mathbb{R}^{N}, X\right)} \leq C\|f\|_{L_{p}\left(\mathbb{R}^{N}, X\right)} .
$$

In particular, $\mathcal{W}_{\psi}$ can be uniquely extended to a bounded linear operator $\mathcal{W}_{\psi, p}$ from $L_{p}\left(\mathbb{R}^{N}, X\right)$ to $L_{p}\left(\mathbb{R}^{N}, Y\right)$, where $Y=L_{q}\left(\Omega, \mu, L_{2}\left(\mathbb{R}_{*}\right)\right)$.

To prove this theorem we will define an operator-valued multiplier function and then apply the Fourier multiplier theorem 2.5. The following lemma will be used to show that our multiplier function satisfies the conditions of Theorem 2.5.

Lemma 3.3. Let $m: \mathbb{R}^{N} \backslash\{0\} \rightarrow \mathbb{C}$ be a measurable function such that $m(\cdot \xi) \in L_{2}\left(\mathbb{R}_{*}\right)$ for each $\xi \in \mathbb{R}^{N}$ with $|\xi|=1$. Then the following hold.

(a) $\xi \mapsto m(\cdot \xi)$ is measurable as a function from $\mathbb{R}^{N} \backslash\{0\}$ to $L_{2}\left(\mathbb{R}_{*}\right)$. Moreover, for $\xi \in \mathbb{R}^{N} \backslash\{0\}$,

$$
M(\xi) x:=m(\cdot \xi) \otimes x, \quad x \in X
$$

defines a bounded linear operator $M(\xi): X \rightarrow Y$. The Banach space adjoint $M(\xi)^{\prime}$ of $M(\xi)$ is given by

$$
M(\xi)^{\prime} y^{\prime}=\left[\omega \mapsto \int_{0}^{\infty} m(t \xi) y^{\prime}(\omega)(t) \frac{d t}{t}\right], \quad y^{\prime} \in Y^{\prime}=L_{q^{\prime}}\left(\Omega, \mu, L_{2}\left(\mathbb{R}_{*}\right)\right) .
$$

(b) If $\{m(\cdot \xi):|\xi|=1\}$ is bounded in $L_{2}\left(\mathbb{R}_{*}\right)$, then $\left\{M(\xi): \xi \in \mathbb{R}^{N} \backslash\{0\}\right\}$ is $R$-bounded in $\mathcal{B}(X, Y)$.

Proof. (a) That $\xi \mapsto m(\cdot \xi)$ is measurable as a function from $\mathbb{R}^{N} \backslash\{0\}$ to $L_{2}\left(\mathbb{R}_{*}\right)$ follows from Pettis' measurability theorem, ${ }^{17}$ since $L_{2}\left(\mathbb{R}_{*}\right)$ is separable and $\xi \mapsto m(\cdot \xi)$ is weakly measurable. The operator $M(\xi)$ is bounded from $X$ to $Y$ since

$$
\|M(\xi) x\|_{Y}=\|m(\cdot \xi)\|_{L_{2}\left(\mathbb{R}_{*}\right)}\|x\|_{X}
$$

for all $x \in X$. Finally, for $x \in X$ and $y^{\prime} \in Y^{\prime}$, we have that

$$
\begin{aligned}
\left\langle M(\xi)^{\prime} y^{\prime}, x\right\rangle_{X} & =\left\langle y^{\prime}, M(\xi) x\right\rangle_{Y}=\int_{\Omega} \overline{\left\langle y^{\prime}(\omega)\right.}|(M(\xi) x)(\omega)\rangle_{L_{2}\left(\mathbb{R}_{*}\right)} d \mu(\omega) \\
& =\int_{\Omega} \overline{\left\langle y^{\prime}(\omega)\right.}|m(\cdot \xi) x(\omega)\rangle_{L_{2}\left(\mathbb{R}_{*}\right)} d \mu(\omega)=\int_{\Omega} \overline{\left\langle y^{\prime}(\omega)\right.}|m(\cdot \xi)\rangle_{L_{2}\left(\mathbb{R}_{*}\right)} x(\omega) d \mu(\omega)
\end{aligned}
$$

and therefore

$$
M(\xi)^{\prime} y^{\prime}=\left[\omega \mapsto\left\langle\overline{y^{\prime}(\omega)} \mid m(\cdot \xi)\right\rangle_{L_{2}\left(\mathbb{R}_{*}\right)}\right]=\left[\omega \mapsto \int_{0}^{\infty} m(t \xi) y^{\prime}(\omega)(t) \frac{d t}{t}\right] .
$$

(b) Observe that the measure $\frac{d t}{t}$ is scale-invariant and therefore, using our assumption,

$$
A:=\sup _{\xi \neq 0}\|m(\cdot \xi)\|_{L_{2}\left(\mathbb{R}_{*}\right)}=\sup _{|\xi|=1}\|m(\cdot \xi)\|_{L_{2}\left(\mathbb{R}_{*}\right)}<\infty .
$$


Now for $n \in \mathbb{N}, \xi_{1}, \ldots \xi_{n} \in \mathbb{R}^{N} \backslash\{0\}$ and $x_{1}, \ldots, x_{n} \in X$ we have by Kahane's inequality and Fubini's theorem

$$
\begin{aligned}
\left(\int_{0}^{1}\left\|\sum_{k=1}^{n} r_{k}(t) M\left(\xi_{k}\right) x_{k}\right\|_{Y}^{2} d t\right)^{1 / 2} & \stackrel{(2)}{\leq} K_{q}\left(\int_{0}^{1}\left\|\sum_{k=1}^{n} r_{k}(t) M\left(\xi_{k}\right) x_{k}\right\|_{Y}^{q} d t\right)^{1 / q} \\
& =K_{q}\left(\int_{0}^{1} \int_{\Omega}\left\|\sum_{k=1}^{n} r_{k}(t) m\left(\cdot \xi_{k}\right) x_{k}(\omega)\right\|_{L_{2}\left(\mathbb{R}_{*}\right)}^{q} d \mu(\omega) d t\right)^{1 / q} \\
\stackrel{\text { (Fubini) }}{=} & K_{q}\left(\int_{\Omega} \int_{0}^{1}\left\|\sum_{k=1}^{n} r_{k}(t) m\left(\cdot \xi_{k}\right) x_{k}(\omega)\right\|_{L_{2}\left(\mathbb{R}_{*}\right)}^{q} d t d \mu(\omega)\right)^{1 / q} .
\end{aligned}
$$

Since $L_{2}\left(\mathbb{R}^{*}\right)$ is a Hilbert space, Kahane's inequality gives

$$
\begin{aligned}
\left(\int_{0}^{1}\left\|\sum_{k=1}^{n} r_{k}(t) m\left(\cdot \xi_{k}\right) x_{k}(\omega)\right\|_{L_{2}\left(\mathbb{R}_{*}\right)}^{q} d t\right)^{1 / q} & \stackrel{(3)}{\leq} K_{q}\left(\sum_{k=1}^{n}\left|x_{k}(\omega)\right|^{2}\left\|m\left(\cdot \xi_{k}\right)\right\|_{L_{2}\left(\mathbb{R}_{*}\right)}^{2}\right)^{1 / 2} \\
& \stackrel{(5)}{\leq} K_{q} A\left(\sum_{k=1}^{n}\left|x_{k}(\omega)\right|^{2}\right)^{1 / 2}
\end{aligned}
$$

for almost every $\omega \in \Omega$. So far we have seen that

$$
\left(\int_{0}^{1}\left\|\sum_{k=1}^{n} r_{k}(t) M\left(\xi_{k}\right) x_{k}\right\|_{Y}^{2} d t\right)^{1 / 2} \leq K_{q}^{2} A\left(\int_{\Omega}\left(\sum_{k=1}^{n}\left|x_{k}(\omega)\right|^{2}\right)^{q / 2} d \mu(\omega)\right)^{1 / q}
$$

But by Kahane's inequality and Fubini's theorem we obtain

$$
\begin{aligned}
&\left(\int_{\Omega}\left(\sum_{k=1}^{n}\left|x_{k}(\omega)\right|^{2}\right)^{q / 2} d \mu(\omega)\right)^{1 / q} \stackrel{(3)}{\leq} K_{q}\left(\int_{\Omega} \int_{0}^{1}\left|\sum_{k=1}^{n} r_{k}(t) x_{k}(\omega)\right|^{q} d t d \mu(\omega)\right)^{1 / q} \\
& \stackrel{\text { (Fubini) }}{=} K_{q}\left(\int_{0}^{1}\left\|\sum_{k=1}^{n} r_{k}(t) x_{k}\right\|_{X}^{q} d t\right)^{1 / q} \\
& \stackrel{(2)}{\leq} \\
& K_{q}^{2}\left(\int_{0}^{1}\left\|\sum_{k=1}^{n} r_{k}(t) x_{k}\right\|_{X}^{2} d t\right)^{1 / 2} .
\end{aligned}
$$

Together we have

$$
\left(\int_{0}^{1}\left\|\sum_{k=1}^{n} r_{k}(t) M\left(\xi_{k}\right) x_{k}\right\|_{Y}^{2} d t\right)^{1 / 2} \leq K_{q}^{4} A\left(\int_{0}^{1}\left\|\sum_{k=1}^{n} r_{k}(t) x_{k}\right\|_{X}^{2} d t\right)^{1 / 2}
$$

But this means that $\left\{M(\xi): \xi \in \mathbb{R}^{N} \backslash\{0\}\right\}$ is R-bounded with R-bound $\leq K_{q}^{4} A$. $\square$

Now we prove Theorem 3.2.

Proof of Theorem 3.2. We define the operator-valued multiplier

$$
M_{\psi}: \mathbb{R}^{N} \backslash\{0\} \rightarrow \mathcal{B}(X, Y), \quad M_{\psi}(\xi) x=\widehat{\psi}(\cdot \xi) \otimes x, \quad \xi \in \mathbb{R}^{N} \backslash\{0\}, x \in X,
$$

and apply the Fourier multiplier theorem 2.5 .

Let $\alpha$ be a multiindex with $|\alpha| \leq l$ and define $m_{\alpha}(\xi)=|\xi|^{|\alpha|}\left(D^{\alpha} \widehat{\psi}\right)(\xi)$. By Lemma 3.3(a) and assumption $(\mathrm{C} 1), \xi \mapsto m_{\alpha}(\cdot \xi)$ is measurable from $\mathbb{R}^{N} \backslash\{0\}$ to $L_{2}\left(\mathbb{R}_{*}\right)$. Moreover, $m_{\alpha}(\cdot \xi)=|\xi|^{|\alpha|}\left(D^{\alpha} m_{0}\right)(\cdot \xi)$. Since $|\xi|^{|\alpha|}\left(D^{\alpha} M_{\psi}\right)(\xi) x=|\cdot \xi|^{|\alpha|}\left(D^{\alpha} m_{0}\right)(\cdot \xi) \otimes x=m_{\alpha}(\cdot \xi) \otimes x$, the distributional derivatives $D^{\alpha} M_{\psi}$ are measurable functions from $\mathbb{R}^{N} \backslash\{0\}$ to $\mathcal{B}(X, Y)$. In addition, Lemma 3.3(b) and assumption (C2) yield that $\left\{|\xi|^{|\alpha|}\left(D^{\alpha} M_{\psi}\right)(\xi)\right.$ : $\left.\xi \in \mathbb{R}^{N} \backslash\{0\}\right\}$ is $R$-bounded for $|\alpha| \leq l$. 
Now Theorem 2.5 implies that $M_{\psi}$ is a Fourier multiplier from $L_{p}\left(\mathbb{R}^{N}, X\right)$ to $L_{p}\left(\mathbb{R}^{N}, Y\right)$. It remains to show that, for $f \in \mathcal{S}\left(\mathbb{R}^{N}, X\right)$ and $s \in \mathbb{R}^{N}$,

$$
\left(\mathcal{W}_{\psi} f\right)(\cdot, s)=(\psi \cdot * f)(s)=(2 \pi)^{-N / 2}\left(\mathcal{F}^{-1}\left[M_{\psi} \widehat{f}\right]\right)(s) \quad \text { almost everywhere in } \mathbb{R}_{*} .
$$

Since $f \in \mathcal{S}\left(\mathbb{R}^{N}, X\right), \sigma \mapsto M_{\psi}(\sigma) \widehat{f}(\sigma)=\widehat{\psi}(\cdot \sigma) \widehat{f}(\sigma)$ is integrable as a function from $\mathbb{R}^{N}$ to $\left.L_{2}\left(\mathbb{R}_{*}, X\right)\right)$. Let $s \in \mathbb{R}^{N}$. By Theorem III.11.17 in [22], the function $t \mapsto\left(\mathcal{F}^{-1}[\widehat{\psi}(t \cdot) \widehat{f}(\cdot)]\right)(s)$ is an element of the equivalence class $\left(\mathcal{F}^{-1}[M \widehat{f}]\right)(s) \in L_{2}\left(\mathbb{R}^{N}, X\right)$. But $\left(\mathcal{F}^{-1}[\widehat{\psi}(t \cdot) \widehat{f}(\cdot)]\right)(s)=(2 \pi)^{N / 2}\left(\psi_{t} * f\right)(s)$. This completes the proof of the theorem. $\square$

TheOREm 3.4. Let $(\Omega, \Sigma, \mu)$ be a $\sigma$-finite measure space, $q \in(1, \infty), X=L_{q}(\Omega, \mu)$, and $Y=L_{q}\left(\Omega, \mu, L_{2}\left(\mathbb{R}_{*}\right)\right)$. If $p \in(1, \infty)$ and $\psi \in L_{2}\left(\mathbb{R}^{N}\right)$ satisfies both $(\mathrm{C} 1)$ and $(\mathrm{C} 2)$, then there is a constant $C$ such that for all $f \in L_{p}\left(\mathbb{R}^{N}, X\right)$,

$$
\frac{1}{C}\|f\|_{L_{p}\left(\mathbb{R}^{N}, X\right)} \leq\left\|\mathcal{W}_{\psi, p} f\right\|_{L_{p}\left(\mathbb{R}^{N}, Y\right)} \leq C\|f\|_{L_{p}\left(\mathbb{R}^{N}, X\right)}
$$

Proof. Observe first, that if $\psi \in L_{2}\left(\mathbb{R}^{N}\right)$ satisfies (C1) and (C2), then $\varphi$, defined by

$$
\widehat{\varphi}(\xi):=\left(\int_{0}^{\infty}|\widehat{\psi}(t \xi)|^{2} \frac{d t}{t}\right)^{-1} \overline{\widehat{\psi}(\xi)}, \quad \xi \in \mathbb{R}^{N}
$$

also satisfies (C1) and (C2). Indeed, using our assumptions on $\psi$ we see immediately that $\varphi \in L_{2}\left(\mathbb{R}^{N}\right)$ and satisfies (C2). To show that $\varphi$ satisfies (C1), we look at $h$, defined by $h(\xi)=\int_{0}^{\infty}|\widehat{\psi}(t \xi)|^{2} \frac{d t}{t}, \xi \in \mathbb{R}^{N} \backslash\{0\}$. By our assumptions on $\psi$, it follows that $h$ and its distributional derivatives of order $\leq l$ are bounded. Moreover, $\frac{1}{h}$ is bounded and therefore has bounded distributional derivatives of order $\leq l$. Using Leibniz' formula and the fact that $h$ is positively homogeneous of degree 0 (i.e. $h(t \xi)=h(\xi)$ for all $t>0$ and all $\xi \in \mathbb{R}^{N} \backslash\{0\}$ ), we obtain that

$$
(t \xi)^{\alpha}\left(D^{\alpha} \widehat{\varphi}\right)(t \xi)=\sum_{\beta \leq \alpha}\left(\begin{array}{l}
\alpha \\
\beta
\end{array}\right) \xi^{\alpha-\beta}\left(D^{\alpha-\beta} \frac{1}{h}\right)(\xi)(t \xi)^{\beta}\left(D^{\beta} \widehat{\psi}\right)(t \xi) .
$$

Hence $\varphi$ satisfies $(\mathrm{C} 2)$.

We define the multiplier function $M_{\varphi}$ by $M_{\varphi}(\xi) x^{\prime}=\widehat{\varphi}(\cdot \xi) \otimes x^{\prime}$. As in the proof of Theorem 3.2 it can be shown that $M_{\varphi}: \mathbb{R}^{N} \backslash\{0\} \rightarrow \mathcal{B}\left(X^{\prime}, Y^{\prime}\right)$ satisfies the assumptions of the multiplier theorem 2.5. Now let $N_{\varphi}(\xi)=M_{\varphi}(\xi)^{\prime}$. Since $X$ and $Y$ both are reflexive, $N_{\varphi}: \mathbb{R}^{N} \backslash\{0\} \rightarrow B(Y, X)$. By Remark 2.6, $N_{\varphi}$ satisfies the assumptions of the multiplier theorem 2.5 and therefore is a Fourier multiplier from $L_{p}\left(\mathbb{R}^{N}, Y\right)$ to $L_{p}\left(\mathbb{R}^{N}, X\right)$. Moreover, by Lemma $3.3(\mathrm{a})$,

$$
N_{\varphi}(\xi) y:=\left[\omega \mapsto \int_{0}^{\infty} \widehat{\varphi}(t \xi) y(\omega)(t) \frac{d t}{t}\right], \quad \xi \in \mathbb{R}^{N} \backslash\{0\}, y \in Y
$$

Now let $M_{\psi}: \mathbb{R}^{N} \backslash\{0\} \rightarrow \mathcal{B}(X, Y)$ as in the proof of Theorem 3.2. Then for all $x^{\prime} \in X^{\prime}$ and $x \in X$,

$$
\left\langle x^{\prime}, N_{\varphi}(\xi) M_{\psi}(\xi) x\right\rangle_{X}=\int_{\Omega} x^{\prime}(\omega) \int_{0}^{\infty} \widehat{\varphi}(t \xi) \widehat{\psi}(t \xi) x(\omega) \frac{d t}{t} d \mu(\omega)=\int_{\Omega} x^{\prime}(\omega) x(\omega) \frac{d t}{t} d \mu(\omega)=\left\langle x^{\prime}, x\right\rangle_{X}
$$

So $N_{\varphi}(\xi) M_{\psi}(\xi)=I d_{X}$ for all $\xi \in \mathbb{R}^{N} \backslash\{0\}$. Therefore

$$
f=\mathcal{F}^{-1}\left(N_{\varphi}(\cdot) M_{\psi}(\cdot) \widehat{f}(\cdot)=\mathcal{F}^{-1}\left(N_{\varphi}(\cdot) \mathcal{F}\left(\mathcal{W}_{\psi} f\right)\right)\right.
$$

Since $N_{\varphi}$ is a Fourier multiplier from $L_{p}\left(\mathbb{R}^{N}, Y\right)$ to $L_{p}\left(\mathbb{R}^{N}, X\right)$, the lower estimate follows. The upper estimate has been shown in Theorem 3.2. So the theorem is proved.

REMARK 3.5. Reconstruction formula

Suppose $\psi, \varphi \in L_{2}(\mathbb{R})$ satisfy $(\mathrm{C} 1)$ and $\int_{\mathbb{R}} \widehat{\varphi}(t \xi) \widehat{\psi}(t \xi) \frac{d t}{t}=1$ for almost all $\xi \in \mathbb{R}^{N} \backslash\{0\}$. Let us denote by $\mathcal{M}_{\varphi}$ the 
Fourier multiplier operator associated with the multiplier function $N_{\varphi}$ defined in (6). Then the proof of Theorem 3.4 yields that $\mathcal{M}_{\varphi}$ is a bounded linear operator from $L_{p}\left(\mathbb{R}^{N}, Y\right)$ to $L_{p}\left(\mathbb{R}^{N}, X\right)$ and that

$$
f=\mathcal{M}_{\varphi} \mathcal{W}_{\psi} f
$$

for all $f \in L_{p}\left(\mathbb{R}^{N}, X\right)$. If $f \in \mathcal{S}\left(\mathbb{R}^{N}, X\right)$, this reconstruction formula can be written as

$$
f=(2 \pi)^{-N} \int_{0}^{\infty} \varphi_{t} * \psi_{t} * f \frac{d t}{t} .
$$

Indeed, for $g \in \mathcal{S}\left(\mathbb{R}^{N}, X^{\prime}\right)$, Fubini's theorem yields

$$
\begin{aligned}
\int_{\mathbb{R}^{N}}\langle g(s), f(s)\rangle_{X} d s & =\int_{\mathbb{R}^{N}} \int_{0}^{\infty} \widehat{\varphi}(t \xi) \widehat{\psi}(t \xi) \frac{d t}{t}\langle\widehat{g}(-\xi), \widehat{f}(\xi)\rangle_{X} d \xi \\
& =\int_{0}^{\infty} \int_{\mathbb{R}^{N}}\langle\widehat{g}(-\xi), \widehat{\varphi}(t \xi) \widehat{\psi}(t \xi) \widehat{f}(\xi)\rangle_{X} d \xi \frac{d t}{t} \\
& =\int_{0}^{\infty} \int_{\mathbb{R}^{N}}\left\langle g(s),(2 \pi)^{-N}\left(\varphi_{t} * \psi_{t} * f\right)(s)\right\rangle_{X} d s \frac{d t}{t} \\
& =\lim _{\substack{\varepsilon \rightarrow 0 \\
R \rightarrow \infty}} \int_{\mathbb{R}^{N}}\left\langle g(s),(2 \pi)^{-N} \int_{\varepsilon}^{R}\left(\varphi_{t} * \psi_{t} * f\right)(s) \frac{d t}{t}\right\rangle_{X} d s
\end{aligned}
$$

(Observe that $\left\|\varphi_{t} * \psi_{t} * f\right\|_{L_{\infty}} \leq \frac{1}{t}\|\varphi\|_{2}\|\psi\|_{2}\|f\|_{1}$.) Now it is not hard to show that for each $f \in \mathcal{S}\left(\mathbb{R}^{N}, X\right)$,

$$
f=(2 \pi)^{-N} \lim _{\substack{\varepsilon \rightarrow 0 \\ R \rightarrow \infty}} \int_{\varepsilon}^{R} \varphi_{t} * \psi_{t} * f \frac{d t}{t},
$$

where the limit is taken in $L_{p}\left(\mathbb{R}^{N}, X\right)$. If we assume in addition that $\varphi, \psi \in L_{1}\left(\mathbb{R}^{N}\right)$, then (7) holds for all $L_{p}\left(\mathbb{R}^{N}, X\right)$.

REMARK 3.6. Classical square functions

Let $P(x)=c_{N}\left(1+|x|^{2}\right)^{-(N+1) / 2}$, where $c_{N}>0$ is such that $\widehat{P}(\xi)=e^{-|\xi|}$. Then $P_{t}(x)=t^{-N} P\left(\frac{x}{t}\right)$ is the Poisson kernel. Let $\psi(x):=\left.\frac{d}{d t} P_{t}(x)\right|_{t=1}$. Then $\psi$ satisfies the conditions $(\mathrm{C} 1)$ and $(\mathrm{C} 2)$. Indeed, $\widehat{\psi}(\xi)=\left.\frac{d}{d t} \widehat{P}_{t}(\xi)\right|_{t=1}=$ $\left.\frac{d}{d t} \widehat{P}(t \xi)\right|_{t=1}=-|\xi| e^{-|\xi|}$. A direct calculation of the derivatives completes the argument.

In the same way one can show that $\varphi(x)=\left.\frac{d}{d t} G_{\sqrt{t}}(x)\right|_{t=1}$ satisfies $(\mathrm{C} 1)$ and $(\mathrm{C} 2)$. Here $G_{\sqrt{t}}$ denotes der Gaussian kernel with $G(x)=e^{-|x|^{2} / 2}$.

With these choices of $\varphi$ and $\psi$ we obtain vector-valued analogues of the g-function of Littlewood-Paley. ${ }^{4,23}$

REMARK 3.7. Further results

In [2], L. Weis and the author prove similar results as Theorem 3.2 and Theorem 3.4 for general UMD spaces

$X$. Here one has to replace the classical square function in (4) by the generalized square function introduced by N. Kalton and L. Weis. ${ }^{7}$

\section{THE SEMI-DISCRETE WAVELET TRANSFORM}

As in the previous section, $X$ denotes the Lebesgue space $L_{q}(\Omega, \mu)$, where $(\Omega, \Sigma, \mu)$ is some $\sigma$-finite measure space and $q \in(1, \infty)$. Now we choose $Y=L_{q}\left(\Omega, \mu, \ell_{2}(\mathbb{Z})\right)$ and assume that $\psi \in L_{2}\left(\mathbb{R}^{N}\right)$ and $a>1$ are such that

(S1) for all $\alpha \in \mathbb{N}_{0}^{N}$ with $|\alpha| \leq l$, the distributional derivatives $D^{\alpha} \widehat{\psi}$ are represented by measurable functions and

$$
C_{\alpha}^{\prime}(\psi):=\sup _{1 \leq|\xi|<a}\left(\sum_{j \in \mathbb{Z}} a^{2 j|\alpha|}\left|\left(D^{\alpha} \widehat{\psi}\right)\left(a^{j} \xi\right)\right|^{2}\right)^{1 / 2}<\infty
$$

$(\mathrm{S} 2) c_{0}^{\prime}(\psi):=\inf _{1 \leq|\xi|<a}\left(\sum_{j \in \mathbb{Z}}\left|\widehat{\psi}\left(a^{j} \xi\right)\right|^{2}\right)^{1 / 2}>0$ 
REMARK 4.1. It is not hard to see that (S1) implies (C1), and (S2) implies (C2). Indeed, suppose (S1) holds. Let $\omega \in \mathbb{C}$ with $|\omega|=1$ and write $\xi=t \omega$. Integrating over $1 \leq t \leq a$ with respect to the measure $\frac{d t}{t}$ yields

$$
\int_{1}^{a} \sum_{j \in \mathbb{Z}} a^{2 j|\alpha|}\left|\left(D^{\alpha} \widehat{\psi}\right)\left(a^{j} t \omega\right)\right|^{2} \frac{d t}{t} \leq C_{\alpha}^{\prime}(\psi)^{2} \log a
$$

But

$$
\begin{gathered}
\int_{1}^{a} \sum_{j \in \mathbb{Z}} a^{2 j|\alpha|}\left|\left(D^{\alpha} \widehat{\psi}\right)\left(a^{j} t \omega\right)\right|^{2} \frac{d t}{t}=\sum_{j \in \mathbb{Z}} \int_{a^{j}}^{a^{j+1}} a^{2 j|\alpha|}\left|\left(D^{\alpha} \widehat{\psi}\right)(t \omega)\right|^{2} \frac{d t}{t} \\
\geq \sum_{j \in \mathbb{Z}} \int_{a^{j}}^{a^{j+1}}\left(\frac{t}{a}\right)^{2|\alpha|}\left|\left(D^{\alpha} \widehat{\psi}\right)(t \omega)\right|^{2} \frac{d t}{t}=a^{-2|\alpha|} \int_{0}^{\infty} t^{2|\alpha|}\left|\left(D^{\alpha} \widehat{\psi}\right)(t \omega)\right|^{2} \frac{d t}{t} .
\end{gathered}
$$

Therefore (C1) holds. For (S2) we proceed in a similar way (see also [24]).

Definition 4.2. Let $a>0$. The semi-discrete wavelet transform $\mathcal{W}_{a, \psi} f$ of a function $f \in \mathcal{S}\left(\mathbb{R}^{N}, X\right)$ with respect to the wavelet $\psi$ is given by

$$
\left(\mathcal{W}_{a, \psi} f\right)(j, s):=\left(\mathcal{W}_{\psi} f\right)\left(a^{j}, s\right), \quad j \in \mathbb{Z}, s \in \mathbb{R}^{N}
$$

Now we can state our main result on the semi-discrete wavelet transform.

THEOREM 4.3. Let $p \in(1, \infty)$.

(a) If $\psi \in L_{2}\left(\mathbb{R}^{N}\right)$ and $a>1$ satisfy (S1), then then there is a constant $C$ such that for all $f \in \mathcal{S}\left(\mathbb{R}^{N}, X\right)$,

$$
\left\|\left(\sum_{j \in \mathbb{Z}}\left|\left(\mathcal{W}_{a, \psi} f\right)(j, \cdot)\right|^{2}\right)^{1 / 2}\right\|_{L_{p}\left(\mathbb{R}^{N}, X\right)} \leq C\|f\|_{L_{p}\left(\mathbb{R}^{N}, X\right)} .
$$

In particular, $\mathcal{W}_{a, \psi}$ can be uniquely extended to a bounded linear operator $\mathcal{W}_{a, \psi, p}$ from $L_{p}\left(\mathbb{R}^{N}, X\right)$ to $L_{p}\left(\mathbb{R}^{N}, Y\right)$, where $Y=L_{q}\left(\Omega, \mu, \ell_{2}(\mathbb{Z})\right)$.

(b) If $\psi \in L_{2}\left(\mathbb{R}^{N}\right)$ and $a>1$ satisfy (S1) and (S2), then there is a constant $C$ such that for all $f \in L_{p}\left(\mathbb{R}^{N}, X\right)$,

$$
\frac{1}{C}\|f\|_{L_{p}\left(\mathbb{R}^{N}, X\right)} \leq\left\|\mathcal{W}_{a, \psi, p} f\right\|_{L_{p}\left(\mathbb{R}^{N}, Y\right)} \leq C\|f\|_{L_{p}\left(\mathbb{R}^{N}, X\right)} .
$$

The proof of this theorem is very similar to the one in the continuous case and will be omitted here.

REMARK 4.4. Reconstruction formula

Suppose that $\psi, \varphi \in L_{2}\left(\mathbb{R}^{N}\right)$ and $a>1$ satisfy (S1) with

$$
\sum_{j \in \mathbb{Z}} \widehat{\varphi}\left(a^{j} \xi\right) \widehat{\psi}\left(a^{j} \xi\right)=1
$$

for almost all $\xi \in \mathbb{R}^{N} \backslash\{0\}$. As in Remark 3.5 we can show that

$$
f=\mathcal{M}_{a, \varphi} \mathcal{W}_{a, \psi} f
$$

for all $f \in L_{p}\left(\mathbb{R}^{N}, X\right)$. If $f \in \mathcal{S}\left(\mathbb{R}^{N}, X\right)$, this reconstruction formula can be written as

$$
f=(2 \pi)^{-N} \sum_{j \in \mathbb{Z}} \varphi_{a^{j}} * \psi_{a^{j}} * f .
$$

If we assume in addition that $\varphi, \psi \in L_{1}\left(\mathbb{R}^{N}\right)$, then (8) holds for all $L_{p}\left(\mathbb{R}^{N}, X\right)$.

REMARK 4.5. Classical square functions

Theorem 4.3 gives a vector-valued version of the fact that for $p \in(1, \infty)$ the homogeneous Triebel-Lizorkin spaces $\dot{F}_{p, 2}^{0}\left(\mathbb{R}^{N}\right)$ are isomorphic to $L_{p}\left(\mathbb{R}^{N}\right) \cdot{ }^{3,5}$ 


\section{ACKNOWLEDGMENTS}

The author wants to thank the Deutsche Forschungsgemeinschaft for supporting her travel to the conference

"Wavelets XI".

\section{REFERENCES}

1. T. Figiel, "On equivalence of some bases to the Haar system in spaces of vector-valued functions," Bull. Polish Acad. Sci. Math. 36(3-4), pp. 119-131, 1988.

2. C. Kaiser and L. Weis, "Wavelet transform for functions with values in UMD spaces." preprint.

3. M. Frazier, B. Jawerth, and G. Weiss, Littlewood-Paley theory and the study of function spaces, vol. 79 of CBMS Regional Conference Series in Mathematics, Published for the Conference Board of the Mathematical Sciences, Washington, DC, 1991.

4. E. M. Stein, Harmonic analysis: real-variable methods, orthogonality, and oscillatory integrals, vol. 43 of Princeton Mathematical Series, Princeton University Press, Princeton, NJ, 1993.

5. H. Triebel, Interpolation theory, function spaces, differential operators, vol. 18 of North-Holland Mathematical Library, North-Holland Publishing Co., Amsterdam, 1978.

6. L. Weis, "Operator-valued Fourier multiplier theorems and maximal $L_{p}$-regularity," Math. Ann. 319(4), pp. $735-758,2001$.

7. N. Kalton and L. Weis, "The $H^{\infty}$-functional calculus and square function estimates." preprint.

8. J. van Neerven and L. Weis, "Stochastic integration in Banach spaces." submitted.

9. J. Peetre, "Sur la transformation de Fourier des fonctions à valeurs vectorielles," Rend. Sem. Mat. Univ. Padova 42, pp. 15-26, 1969.

10. S. Kwapień, "Isomorphic characterizations of inner product spaces by orthogonal series with vector valued coefficients," Studia Math. 44, pp. 583-595, 1972.

11. H. Amann, Linear and quasilinear parabolic problems. Vol. I, vol. 89 of Monographs in Mathematics, Birkhäuser Boston Inc., Boston, MA, 1995.

12. D. J. Aldous, "Unconditional bases and martingales in $L_{p}(F)$," Math. Proc. Cambridge Philos. Soc. 85(1), pp. 117-123, 1979.

13. P. Enflo, "Banach spaces which can be given an equivalent uniformly convex norm," in Proceedings of the International Symposium on Partial Differential Equations and the Geometry of Normed Linear Spaces (Jerusalem, 1972), Israel J. Math. 13, pp. 281-288, 1972.

14. J. Diestel, H. Jarchow, and A. Tonge, Absolutely summing operators, vol. 43 of Cambridge Studies in Advanced Mathematics, Cambridge University Press, Cambridge, 1995.

15. J. Bourgain, "A Hausdorff-Young inequality for B-convex Banach spaces," Pacific J. Math. 101(2), pp. 255$262,1982$.

16. J. Bourgain, "Vector-valued Hausdorff-Young inequalities and applications," in Geometric aspects of functional analysis (1986/87), Lecture Notes in Math. 1317, pp. 239-249, Springer, Berlin, 1988.

17. J. Diestel and J. J. Uhl, Jr., Vector measures, American Mathematical Society, Providence, R.I., 1977.

18. M. Girardi and L. Weis, "Operator-valued Fourier multiplier theorems on Besov spaces," Math. Nachr. 251, pp. 34-51, 2003.

19. M. Girardi and L. Weis, "Operator-valued Fourier multiplier theorems on $L_{p}(X)$ and geometry of Banach spaces," J. Funct. Anal. 204(2), pp. 320-354, 2003.

20. J. Bergh and J. Löfström, Interpolation spaces. An introduction, Springer-Verlag, Berlin, 1976.

21. H. Witvliet, Unconditional Schauder decompositions and multiplier theorems. PhD thesis, Technische Universiteit Delft, 2000.

22. N. Dunford and J. T. Schwartz, Linear operators. Part I, Wiley Classics Library, John Wiley \& Sons Inc., New York, 1988.

23. H. Triebel, Theory of function spaces. II, vol. 84 of Monographs in Mathematics, Birkhäuser Verlag, Basel, 1992.

24. C. K. Chui and X. L. Shi, "Inequalities of Littlewood-Paley type for frames and wavelets," SIAM J. Math. Anal. 24(1), pp. 263-277, 1993. 